\title{
Projective synchronization for 4D hyperchaotic system based on adaptive nonlinear control strategy
}

\author{
Zaidoon Sh. Al-Talib, Saad Fawzi AL-Azzawi \\ Department of Mathematics, University of Mosul, Iraq
}

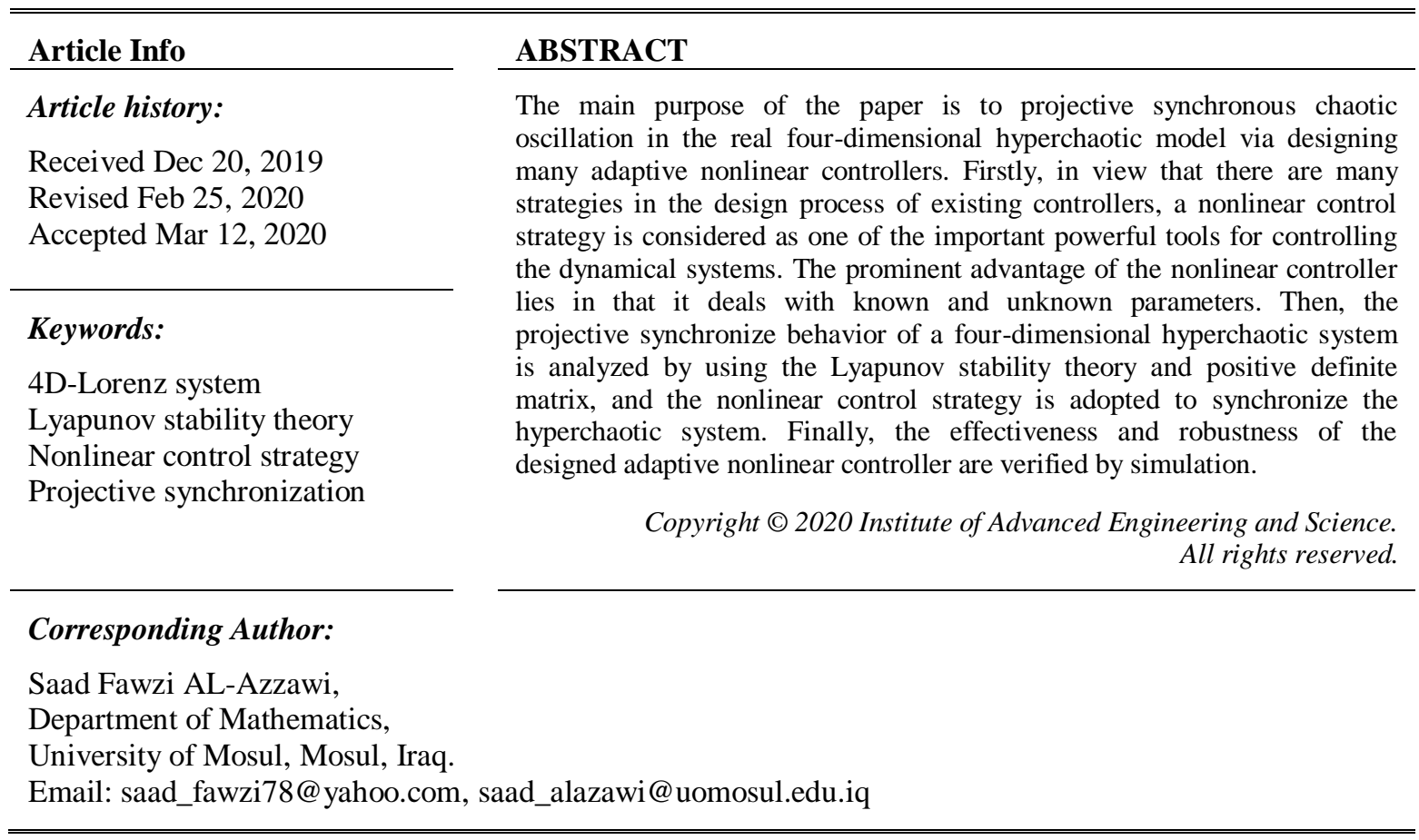

\section{INTRODUCTION}

In the field of the nonlinear dynamical system, Lorenz system is the first classical three-dimensional chaotic system which discovers in 1963, it followed that several attempts to create another chaotic system such as Chen system (1999), Lu“ system (2002), Liu system (2004), Pan system (2009) and so on [1]. In addition, generate higher-dimensional hyperchaotic systems. The hyperchaotic system was firstly introduced in 1979 by Rossler, this system is one of a high-dimensional and strongly coupled nonlinear dynamical system, often exhibits complex nonlinear dynamic behaviors and contains at least two positive Lyapunov exponents [2-3].

In recent years, the control and synchronization of the aforementioned systems have gained enough attention from scholars due to its potential applications in engineering [4-5], electrical circuits, biological systems [6-7], secure communication [8]. It is well known that the idea of synchronization was firstly proposed by [8-11]. The concept of synchronization has been extended to the scope, such as complete synchronization (CS) [1, 3, 8, 12], anti-synchronization (AS) [10, 13], generalized synchronization [14], projective synchronization [15-17] and so on. Various strategies have been introduced until now to realize the stabilization of error dynamic systems, including active control, adaptive control, nonlinear control [18-22], linear feedback control [16]. Among many of these control strategies, nonlinear control has attracted many scholars' attention because of its simplicity, reliability, and effectiveness and widely used as one powerful strategy for synchronization of a different class of nonlinear dynamical systems [23-26]. However, the designed control input must depend on the controlled system functions according to the conventional nonlinear control. In order to simplify the control input, adaptive nonlinear control can be designed to make its control inputs easy to be realized synchronization.

In any control system applications, the main objective is to design a controller for a good performance and robustness. To ensure that the designed adaptive nonlinear controller has a good control 
effect, a nonlinear controller is designed for a controlled system based on the Lyapunov stability theory with known and unknown parameters. Then, the designed controllers are used to synchronize the hyperchaotic system. These findings may be of significance in understanding and controlling problems in modern society. Simulation results verify the effectiveness and robustness of the proposed control scheme.

\section{DESCRIPTION OF 4-D LORENZ SYSTEM}

The Lorenz chaotic system is the first famous model of 3-D chaotic behavior which modeled into a four-dimensional by Guangyun et al. In 2017 [27] via state feedback control which consists of two nonlinearity and seven parameters. The new system is depicted as follows.

$$
\left\{\begin{array}{c}
\dot{x}=a(y-x)-f w \\
\dot{y}=x z-q y \\
\dot{z}=b-x y-c z \\
\dot{w}=r y-d w
\end{array}\right.
$$

Where $x, y, z$, and $w$ are state variables and $a, b, c, d, r, f, q$ are positive parameters the system (1). When the parameters taken the values $a=5, b=20, c=1, d=0.1, r=0.1, f=20.6, q=1$, the system (1) exhibits hyperchaotic behavior due to it has two positive Lyapunov exponents such as $L E_{1}=0.24, L E_{2}=0.23$. Figure 1 show attractor the system (1).

(a)

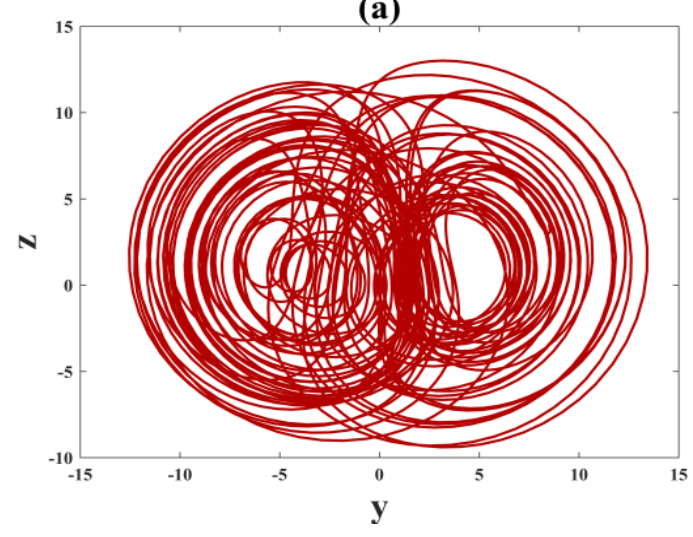

(c)

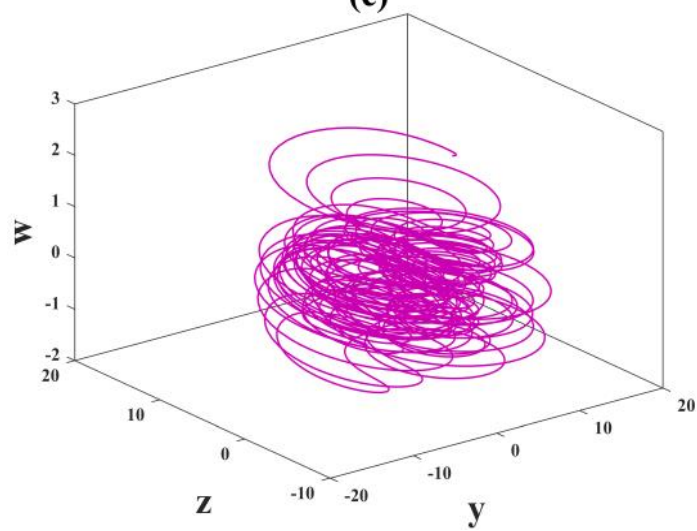

(b)

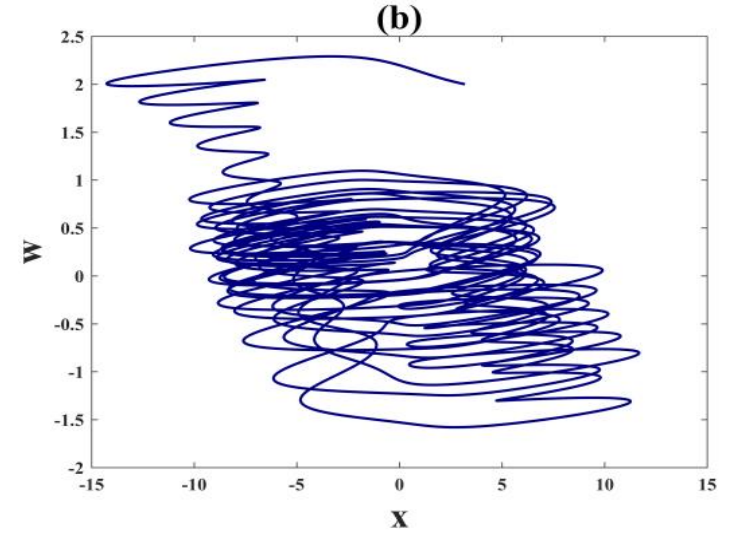

(d)

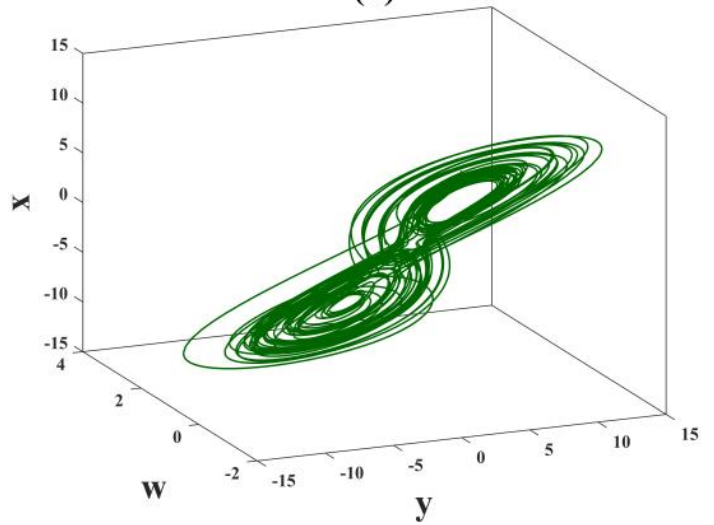

Figure 1. Show attractor the system (1) a: y, z-plane, b: x, w-plane, c: y, z, w-space, d: y, w, x-space.

\section{THE PROJECTIVE SYNCHRONIZATION OF 4-D LORENZ SYSTEM}

In this section, the projective synchronization between two identical 4-D hyperchaotic Lorenz system is implemented via nonlinear control strategy, the drive and response systems are given by (2) and (3) respectively as: 


$$
\begin{gathered}
\left\{\begin{array}{c}
\dot{x}_{1}=a\left(y_{1}-x_{1}\right)-f w_{1} \\
\dot{y}_{1}=x_{1} z_{1}-q y_{1} \\
\dot{z}_{1}=b-x_{1} y_{1}-c z_{1} \\
\dot{w}_{1}=r y_{1}-d w_{1}
\end{array}\right. \\
\left\{\begin{array}{c}
\dot{x}_{2}=a\left(y_{2}-x_{2}\right)-f w_{2}+u_{1} \\
\dot{y}_{2}=x_{2} z_{2}-q y_{2}+u_{2} \\
\dot{z}_{2}=b-x_{2} y_{2}-c z_{2}+u_{3} \\
\dot{w}_{2}=r y_{2}-d w_{2}+u_{4}
\end{array}\right.
\end{gathered}
$$

Where $\left(x_{i}, y_{i}, z_{i}, w_{i}\right) \in R^{4}, U=\left[u_{1}, u_{2}, u_{3}, u_{4}\right]^{T}$ Represents control input to be designed, by using the following law.

$$
\left\{\begin{array}{l}
\dot{e}_{1}=x_{2}-\alpha_{1} x_{1} \\
\dot{e}_{2}=y_{2}-\alpha_{2} y_{1} \\
\dot{e}_{3}=z_{2}-\alpha_{3} z_{1} \\
\dot{e}_{4}=w_{2}-\alpha_{4} w_{1}
\end{array}\right.
$$

For $\forall \alpha_{i}=1, i=1,2,3,4$, the corresponding error dynamical system is given as:

$$
\left\{\begin{array}{c}
\dot{e}_{1}=a\left(e_{2}-e_{1}\right)-f e_{4}+u_{1} \\
\dot{e}_{2}=-q e_{2}+e_{1} z_{1}+e_{3} x_{1}+e_{1} e_{3}+u_{2} \\
\dot{e}_{3}=-c e_{3}-e_{1} y_{1}-e_{2} x_{1}-e_{1} e_{2}+u_{3} \\
\dot{e}_{4}=-d e_{4}+r e_{2}+u_{4}
\end{array}\right.
$$

\subsection{Projective synchronization with unknown parameters}

In this subsection, we design adaptive nonlinear control to make the system (3) synchronizes with the system (2) with unknown parameters.

Theorem 1. If adaptive nonlinear control is selected as follows:

$$
\left\{\begin{array}{c}
u_{1}=0 \\
u_{2}=-a e_{1}-e_{1} z_{1}-r e_{4} \\
u_{3}=e_{1} y_{1} \\
u_{4}=f e_{1}
\end{array}\right.
$$

Then the system (3) follows the trace of the system (2), which means that the projective synchronization can be achieved by the designed controller (6).

Proof: Insert the controller (6) in the error dynamical system (5), we obtain

$$
\left\{\begin{array}{c}
\dot{e}_{1}=a\left(e_{2}-e_{1}\right)-f e_{4} \\
\dot{e}_{2}=-q e_{2}+e_{3} x_{1}+e_{1} e_{3}-a e_{1}-r e_{4} \\
\dot{e}_{3}=-c e_{3}-e_{2} x_{1}-e_{1} e_{2} \\
\dot{e}_{4}=-d e_{4}+r e_{2}+f e_{1}
\end{array}\right.
$$

To achieve projective synchronization for the system (7) theoretically, select the Lyapunov function as the following:

$$
V(e)=e^{T} P e, P=\left[\begin{array}{cccc}
\frac{1}{2} & 0 & 0 & 0 \\
0 & \frac{1}{2} & 0 & 0 \\
0 & 0 & \frac{1}{2} & 0 \\
0 & 0 & 0 & \frac{1}{2}
\end{array}\right]
$$

The time derivative of the Lyapunov function is:

$$
\dot{V}(e)=-a e_{1}^{2}-q e_{2}^{2}-c e_{3}^{2}-d e_{4}^{2}=-e^{T} Q e
$$


Herein $Q=\left[\begin{array}{llll}a & 0 & 0 & 0 \\ 0 & q & 0 & 0 \\ 0 & 0 & c & 0 \\ 0 & 0 & 0 & d\end{array}\right]$

So, every diagonal matrix with positive diagonal elements is a positive definite. Therefore, $\dot{V}(e)$ is also a positive definite function. The projective synchronization is realized between system (3) and system (2) based on the proposed control (6). This theoretical result is illustrated by numerical simulation as shown in Figure 2.
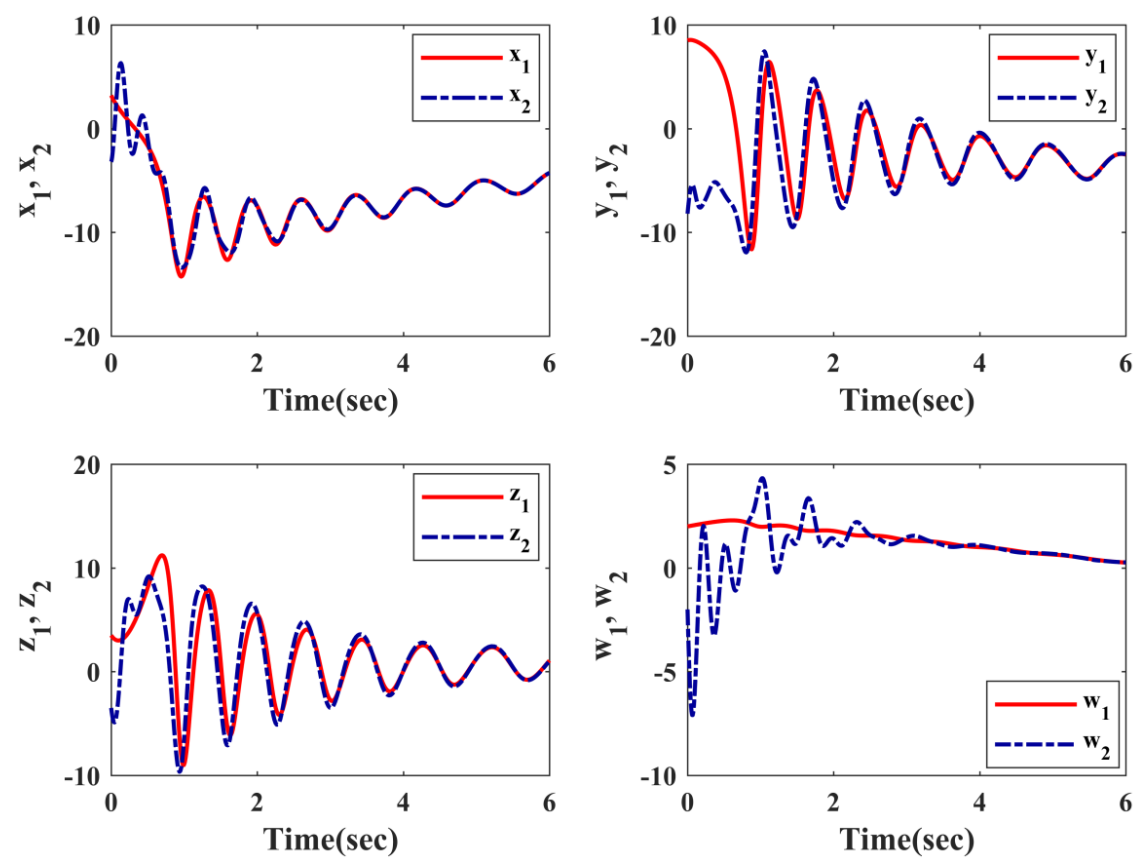

Figure 2. Projective synchronizes for the system (5) with controller (6)

\subsection{Projective synchronization with known parameters}

In the previous subsection (subsection 3.1), the projective synchronization with unknown parameters was performed theoretically and numerically without any problems. This subsection will be considered the projective synchronization with known parameters and note that there some problems in this case. And how we deal with these problems and what are treating? The following theory answer this question.

Theorem 2. If the adaptive nonlinear controller is designed as follows.

$$
\left\{\begin{array}{c}
u_{1}=-e_{2} z_{1}+e_{3} y_{1} \\
u_{2}=-b e_{1}-r e_{4} \\
u_{3}=0 \\
u_{4}=f e_{1}
\end{array}\right.
$$

Then system (5) will be projective synchronized.

Proof: When substituting the controllers (9) in the system (5), we get

$$
\left\{\begin{array}{c}
\dot{e}_{1}=a\left(e_{2}-e_{1}\right)-f e_{4}-e_{2} z_{1}+e_{3} y_{1} \\
\dot{e}_{2}=-q e_{2}+e_{1} z_{1}+e_{3} x_{1}+e_{1} e_{3}-b e_{1}-r e_{4} \\
\dot{e}_{3}=-c e_{3}-e_{1} y_{1}-e_{2} x_{1}-e_{1} e_{2} \\
\dot{e}_{4}=-d e_{4}+r e_{2}+f e_{1}
\end{array}\right.
$$

Based on theoretically analysis, the Lyapunov function and its derivative yields the (11) and (12), respectively. 


$$
\begin{aligned}
& V(e)=\frac{1}{2} \sum_{i=1}^{4} e^{2} \\
& \dot{V}(e)=-a e_{1}^{2}-q e_{2}^{2}-c e_{3}^{2}-d e_{4}^{2}+(a-b) e_{1} e_{2}=-e^{T} Q_{1} e
\end{aligned}
$$

Where

$$
Q_{1}=\left[\begin{array}{cccc}
a & -\frac{(a-b)}{2} & 0 & 0 \\
-\frac{(a-b)}{2} & q & 0 & 0 \\
0 & 0 & c & 0 \\
0 & 0 & 0 & d
\end{array}\right]
$$

To ensure that the system (10) is asymptotically stable, the symmetric matrix $Q$ should be positive definite if the following conditions must hold:

$$
\left\{\begin{array}{c}
a, c, d>0 \\
4 a q>(a-b)^{2}
\end{array}\right.
$$

But, the second inequality of (13) is incorrect when the parameters are known. So, this control is failing. For this reason, we attempt to overcome this problem via two treatments:

a) Modifying the control only.

b) Modifying the control with Lyapunov function.

First treatment: The first treatment is by modifying the control itself through the modifying the first equation of control (9) i.e., add the term $\left(3 a e_{2}\right)$. we obtain the following control:

$$
\left\{\begin{array}{c}
u_{1}=-e_{2} z_{1}+e_{3} y_{1}+3 a e_{2} \\
u_{2}=-b e_{1}-r e_{4} \\
u_{3}=0 \\
u_{4}=f e_{1}
\end{array}\right.
$$

And the error dynamical system (5) with this control is becoming,

$$
\left\{\begin{array}{c}
\dot{e}_{1}=a\left(e_{2}-e_{1}\right)-f e_{4}-e_{2} z_{1}+e_{3} y_{1}+3 a e_{2} \\
\dot{e}_{2}=-q e_{2}+e_{1} z_{1}+e_{3} x_{1}+e_{1} e_{3}-b e_{1}-r e_{4} \\
\dot{e}_{3}=-c e_{3}-e_{1} y_{1}-e_{2} x_{1}-e_{1} e_{2} \\
\dot{e}_{4}=-d e_{4}+r e_{2}+f e_{1}
\end{array}\right.
$$

Theoretically, based on Lyapunov function (11), the derivative for this function yield:

$$
\begin{aligned}
& \dot{V}(e)=-a e_{1}^{2}-q e_{2}^{2}-c e_{3}^{2}-d e_{4}^{2}+(4 a-b) e_{1} e_{2}=-e^{T} Q_{2} e \\
& Q_{2}=\left[\begin{array}{cccc}
a & \frac{(b-4 a)}{2} & 0 & 0 \\
\frac{(b-4 a)}{2} & q & 0 & 0 \\
0 & 0 & c & 0 \\
0 & 0 & 0 & d
\end{array}\right]
\end{aligned}
$$

Substitute the value of parameters in above matrix we get

$$
Q_{2}=\left[\begin{array}{cccc}
5 & 0 & 0 & 0 \\
0 & 1 & 0 & 0 \\
0 & 0 & 1 & 0 \\
0 & 0 & 0 & \frac{1}{10}
\end{array}\right]
$$

So,

$$
\dot{V}(e)=-5 e_{1}^{2}-e_{2}^{2}-e_{3}^{2}-0.1 e_{4}^{2}
$$


Then $\dot{V}(e)<0$. Therefore, we succeed to achieve control for the system (5) after we are modifying control (9). This theoretical result is illustrated by numerical simulation as shown in Figure 3.
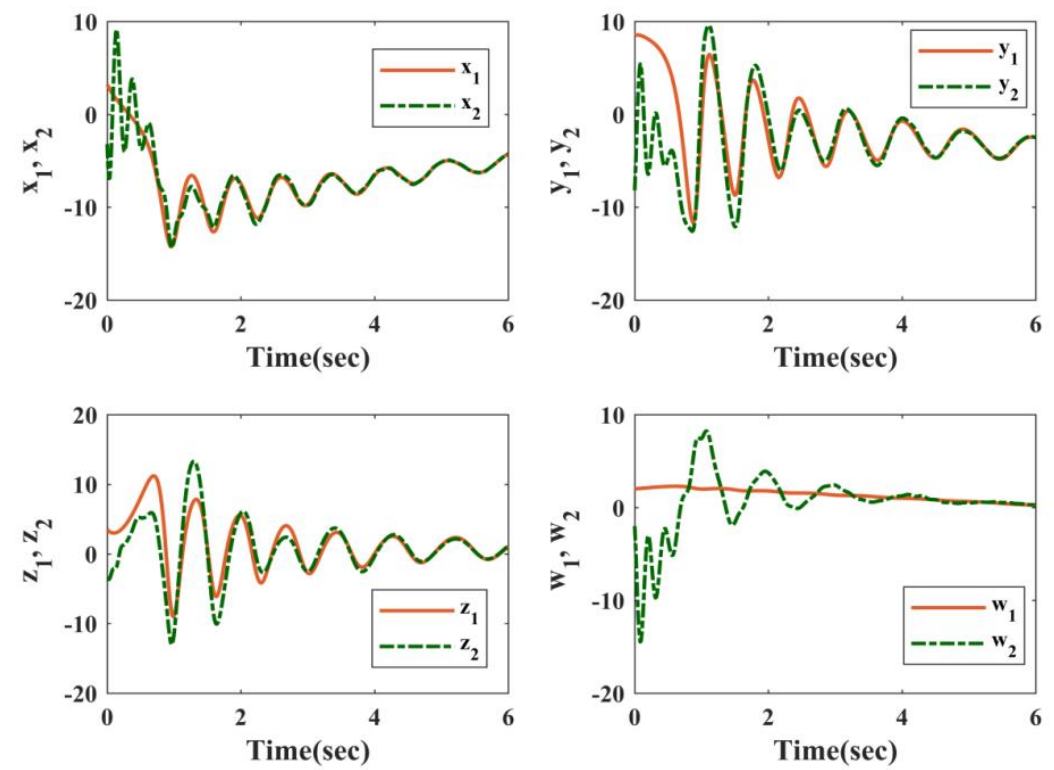

Figure 3. Projective synchronizes for the system (5) with the controller (14)

Second treatment: The second treatment is by modifying both control and Lyapunov function via modifying the second equation for control (5) i.e., replace the term $\left(-b e_{1}\right)$ be $\left(-\frac{1}{4} b e_{1}\right)$. In other words, multiply the term $\left(-b e_{1}\right)$ be the constant $\left(\frac{1}{4}\right)$, and the same time modifying the matrix $P$ in $(8)$.

$$
\left\{\begin{array}{c}
u_{1}=-e_{2} z_{1}+e_{3} y_{1} \\
u_{2}=-\frac{1}{4} b e_{1}-r e_{4} \\
u_{3}=0 \\
u_{4}=f e_{1}
\end{array}\right.
$$

And the error dynamical system (5) with this control is becomes,

$$
\left\{\begin{array}{c}
\dot{e}_{1}=a\left(e_{2}-e_{1}\right)-f e_{4}-e_{2} z_{1}+e_{3} y_{1} \\
\dot{e}_{2}=-q e_{2}+e_{1} z_{1}+e_{3} x_{1}+e_{1} e_{3}-\frac{1}{4} b e_{1}-r e_{4} \\
\dot{e}_{3}=-c e_{3}-e_{1} y_{1}-e_{2} x_{1}-e_{1} e_{2} \\
\dot{e}_{4}=-d e_{4}+r e_{2}+f e_{1}
\end{array}\right.
$$

Now, according to Lyapunov's second method, the Lyapunov function will be modified as follows:

$$
V(e)=e^{T} P_{1} e=\frac{1}{2}\left[4 e_{1}^{2}+4 e_{2}^{2}+4 e_{3}^{2}+4 e_{4}^{2}\right]
$$

Where the diagonal matrix $P_{1}$ modified as:

$$
P_{1}=\left[\begin{array}{llll}
2 & 0 & 0 & 0 \\
0 & 2 & 0 & 0 \\
0 & 0 & 2 & 0 \\
0 & 0 & 0 & 2
\end{array}\right]
$$

Consequently, the derivative of Lyapunov function becomes:

$$
\dot{V}(e)=-4 a e_{1}^{2}-4 q e_{2}^{2}-4 c e_{3}^{2}-4 d e_{4}^{2}+(4 a-b) e_{1} e_{2}=-e^{T} Q_{3} e
$$


when $Q_{3}=\left[\begin{array}{cccc}4 a & -\frac{(4 a-b)}{2} & 0 & 0 \\ -\frac{(4 a-b)}{2} & 4 q & 0 & 0 \\ 0 & 0 & 4 c & 0 \\ 0 & 0 & 0 & 4 d\end{array}\right]=\left[\begin{array}{cccc}20 & 0 & 0 & 0 \\ 0 & 4 & 0 & 0 \\ 0 & 0 & 4 & 0 \\ 0 & 0 & 0 & 0.4\end{array}\right]$.

So, $Q_{3}$ is positive definite. Therefore, we succeed to achieve control for the system (9) after we update modifies the control with Lyapunov function theoretically. Figure 4 shows these results numerically.
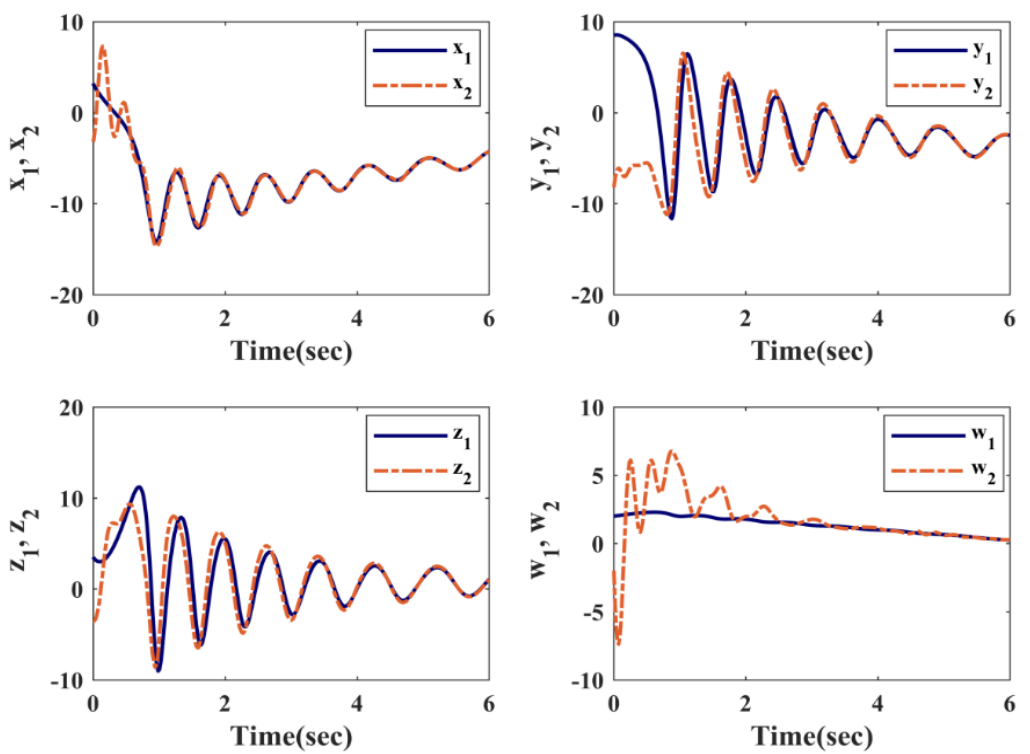

Figure 4. Projective synchronizes for the system (5) with the controller (16)

\section{CONCLUSION}

In this paper, the projection synchronization between two identical four-dimensional hyperchaotic systems is dealt with. Two control with known and unknown parameters are proposed based on nonlinear control strategies, instead of the sliding mode control which is widely used in literature. In order to obtain an appropriate controller, many modifying on control are achieved through control itself and the Lyapunov function. As a result of two controllers based on theoretical analysis and numerical simulations, it is observed that two proposed control methods in the paper produce better outcomes than the sliding mode control.

\section{ACKNOWLEDGEMENTS}

The authors are very grateful to the University of Mosul/College of Computer Sciences and Mathematics for their provided facilities, which helped to improve the quality of this work.

\section{REFERENCES}

[1] H. K. Chen, "Global chaos synchronization of new chaotic systems via nonlinear control," Chaos, Solitons \& Fractals, vol. 23, no. 4, pp. 1245-1251, Feb 2005.

[2] S. F. Al-Azzawi, "Stability and bifurcation of pan chaotic system by using routh-hurwitz and gardan method," Applied Mathematics and Computation, vol. 219, no. 3, pp. 1144-1152, Oct 2012.

[3] J. H. Park, "Chaos synchronization of a chaotic system via nonlinear control," Chaos Solitons Fractals, vol. 25, no. 3, pp. 579-584, 2005.

[4] K. A. Abed and A. A. Ahmad, "The best parameters selection using PSO algorithm to solving for ito system by new iterative technique," Indonesian Journal of Electrical Engineering and Computer Science, vol. 18, no. 3, pp. 1638-1645, June 2020.

[5] A. F., Qasim and B. J. Salim, "Application new iterative method for solving modified korteweg-devries (MKdV) system from three equations," Journal of Advanced Research in Dynamical and Control Systems, vol. 11, no. 3, pp.1-7, 2019. 
[6] Z. N. Al-Khateeb, M. F. Jader, "Encryption and hiding text using DNA coding and hyperchaotic system," Indonesian Journal of Electrical Engineering and Computer Science, vol. 19, no. 2, Aug 2020.

[7] M. A. A. Alhafedh and O. S. Qasim, "Two-stage gene selection in microarray dataset using fuzzy mutual information and binary particle swarm optimization," Indian Journal of Forensic Medicine \& Toxicology, vol. 13, no. 4, pp. 1162-1171, 2019.

[8] M. E. Sahin, et al. "Application and modeling of a novel 4D memristive chaotic system for communication systems," Circuits, Systems, and Signal Processing, Jan 2020.

[9] S. F. Al-Azzawi and M. M. Aziz, "Chaos synchronization of nonlinear dynamical systems via a novel analytical approach,” Alexandria Engineering Journal, vol. 57, no. 4, pp. 3493-3500, Dec 2018.

[10] M. M. Aziz and S. F. Al-Azzawi, "Anti-synchronization of nonlinear dynamical systems based on gardano's method," Optik, vol. 134, pp. 109-120, Apr 2017.

[11] M. Srivastava, et al., "Anti-synchronization between identical and non-identical fractional-order chaotic systems using active control method," Nonlinear Dynamics, vol. 76, no. 2, pp. 905-914, Apr 2014.

[12] M. M. Aziz and S. F. Al-Azzawi, "Hybrid chaos synchronization between two different hyperchaotic systems via two approaches," Optik, vol. 138, pp. 328-340, Jun 2017.

[13] S. Y. Al-hayali and S. F. AL-Azzawi, "An optimal control for complete synchronization of 4D rabinovich hyperchaotic systems," TELKOMNIKA (Telecommunication, Computing, Electronics and Control), vol. 18, no 2, pp. 994-1000, Apr 2020.

[14] S. Y. Al-hayali and S. F. AL-Azzawi, "An optimal nonlinear control for anti-synchronization of rabinovich hyperchaotic system," Indonesian Journal of Electrical Engineering and Computer Science, vol. 19, no. 1, pp. 380-387, Jul 2020.

[15] Y. W. Wang and Z. H. Guan, "Generalized synchronization of continuous chaotic system," Chaos, Solitons \& Fractals, vol. 27, no.1, pp. 97-101, Jan 2006.

[16] Y. D. Chu, et al., "Full state hybrid projective synchronization in hyperchaotic systems," Chaos, Solitons \& Fractals, vol. 42, no. 3, pp. 1502-1510, Nov 2009.

[17] A. S. Al-Obeidi and S. F. Al-Azzawi, "Projective synchronization for a class of 6-D hyperchaotic lorenz system," Indonesian Journal of Electrical Engineering and Computer Science, vol. 16, no. 2, pp. 692-700, Nov 2019.

[18] Z. Sh. Al-Talib and S. F. AL-Azzawi, "Projective and hybrid projective synchronization of 4-D hyperchaotic system via nonlinear controller strategy," TELKOMNIKA (Telecommunication, Computing, Electronics and Control), vol. 18, no 2, pp. 1012-1020, April 2020.

[19] C. Li and J. Yan, "Generalized projective synchronization of chaos: The cascade synchronization approach," Chaos, Solitons \& Fractals, vol. 30, no. 1, pp. 140-146, Oct 2006.

[20] S. Vaidyanathan, et al., "A new chaotic system with axe-shaped equilibrium, its circuit implementation and adaptive synchronization," Archives of Control Sciences, vol. 28, no. 3, pp. 443-462, 2018.

[21] M. T. Yassen, "Chaos synchronization between two different chaotic systems using active control," Chaos, Solitons \& Fractals, vol. 23, no. 1, pp. 131-140, Jan 2005.

[22] A. S. Al-Obeidi and S. F. Al-Azzawi, "Complete synchronization of a novel 6-D hyperchaotic lorenz system with known parameters," International Journal of Engineering \& Technology, vol. 7, no.4, pp. 5345-5349, 2018.

[23] A. S. Al-Obeidi and S. F. Al-Azzawi, "Chaos synchronization of a class 6-D hyperchaotic lorenz system," Modelling, Measurement and Control B, vol. 88, no.1, pp. 17-22, Sep 2019.

[24] M. T. Yassen, "Controlling chaos and synchronization for new chaotic system using linear feedback control," Chaos, Solitons \& Fractals, vol. 26, no. 3, pp. 913-920, Nov 2005.

[25] S. F. Al-Azzawi and M. M. Aziz, "Strategies of linear feedback control and its classification," TELKOMNIKA (Telecommunication, Computing, Electronics and Control), vol. 17, no. 4, pp. 1931-1940, Aug 2019.

[26] A. S. Al-Obeidi and S. F. Al-Azzawi, "Chaos synchronization in a 6-D hyperchaotic system with selfexcited attractor," TELKOMNIKA (Telecommunication, Computing, Electronics and Control), vol. 18, no 3, pp. 1483-1490, Jun 2020.

[27] G. Zhang, et al., "On the dynamics of new 4d lorenz-type chaos systems," Advances in Difference Equations, vol. 2017, no. 1, pp. 1-13, Dec 2017. 